\title{
Hémangioendothéliome intravasculaire végétant lingual : à propos d'un cas
}

\author{
C. Nayl (Vannes), M. Fenelon (Bordeaux), S. Catros (Bordeaux), J.C. Fricain (Bordeaux)
}

L'hémangioendothéliome intravasculaire végétant ou tumeur de Masson est une pathologie tumorale bénigne secondaire à la prolifération réactive de cellules endothéliales papillomateuses liées à un thrombus (Masson P., 1923). Il s'agit d'une tumeur vasculaire relativement rare qui représente $2 \%$ des tumeurs vasculaires des tissus cutanés et sous cutanés. Peu d'études rapportent sa localisation endobuccale, cependant il existe de nombreux cas dans la littérature décrivant sa prédilection pour la région cervicocranio-faciale (Lancaster et al, 1998). Un patient âgé de 71 ans, sans antécédents médico-chirurgicaux notables, était adressé à la consultation spécialisée de pathologie de la muqueuse buccale pour une tuméfaction de la face dorsale de la langue de découverte fortuite. Il s'agissait d'une lésion nodulaire d'un centimètre de grand axe, de coloration violacé et indolore. À la palpation on ne retrouvait pas de battement. Une exérèse chirurgicale au laser diode a été réalisée sous anesthésie locale. Une incision jusqu'aux plans musculaires au niveau desquels s'insinuait la tumeur a été réalisée. Les suites opératoires ont été simples. Le patient a été revu à 3 mois sans signe de récidive. L'analyse anatomopathologique mettait en évidence un hémangioendothéliome intravasculaire végétant. La cicatrisation à 1 mois post-opératoire était satisfaisante. L'hémangioendothéliome intravasculaire végétant est une tumeur vasculaire bénigne dont l'étiopathogénie reste encore discutée. Trois formes sont décrites ; la forme primaire apparaissant dans des vaisseaux distendus, la forme secondaire à des lésions vasculaire préexistantes, et la forme extravasculaire (Bologna-Molina et al, 2010). Cependant, il a été observé que la majorité des cas d'hémangioendothéliome intravasculaire végétant, quel que soit leur type, sont associés à un thrombus (Korkolis et al, 2005). L'hémangioendothéliome intravasculaire végétant est une pathologie rare souvent confondue avec une malformation vasculaire ou une lésion maligne telle que l'angiosarcome. L'établissement d'un diagnostic positif est essentiel pour écarter le diagnostic différentiel d'angiosarcome et éviter toute chirurgie inutilement invasive pour le patient. La prise en charge de l'hémangioendothéliome intravasculaire végétant repose sur son exérèse. 